\title{
Proofs by Reductio ad Impossibile in Aristotle's Prior Analytics ${ }^{1}$
}

\author{
Antônio Mesquita Neto
}

\begin{abstract}
This paper aims at clarifying the procedure of proofs by reductio ad impossibile in Aristotle's Prior Analytics, especially elucidating what can be taken as impossibility in such proofs. Traditional interpretation has it that the impossibility in Aristotle's reductio proofs must be a contradiction. I argue for an alternative interpretation according to which both contrarieties and contradictions are suitable as the impossibility required by the proofs in question. I also present a definition of proof by reductio ad impossibile in accordance with the alternative interpretation.
\end{abstract}

A first distinction to be made, in order to introduce the main point of this paper, is between syllogism and proof. Such a distinction is not easily made nor is it free of controversy. Consider the following preliminary distinction. Let syllogism be an inference of a conclusion from a set of premises that satisfies Aristotle's definition of syllogism in Prior Analytics I $1(24 \mathrm{~b} 18-22)^{2}$. Thus, a proof of that inference is also an inference of the same conclusion from the same set of premises that satisfies Aristotle's definition, but an inference that contains additional steps between the premises and the conclusion in order to show that the first inference is syllogistic ${ }^{3}$. Moreover, consider the following examples of each part of the distinction: an example of syllogism is the

\footnotetext{
${ }^{1}$ I would like to thank Professor Wellington Damasceno (UFG), Professor Mateus Ferreira (UEM), Professor Vitor Bragança (UFG), Cristiane Martins (UFG) and an anonymous referee for their helpful comments on drafts of this paper.

${ }^{2}$ Scholars have been debating over Aristotle's definition of syllogism. Its interpretation is controversial and its study is not under the scope of this paper. For further information and references on Aristotle's definition of syllogism, see Smith 1989, p. 109-110 and Striker 2009, p. $78-82$.

${ }^{3}$ Aristotle's proofs in the Prior Analytics aim at showing that an inference is syllogistic. Whether being syllogistic is the same as being valid is a matter beyond the scope of this paper, but that should not be taken for granted. There are passages that suggest that being syllogistic requires more than mere validity. One of these passages is the aforementioned definition of syllogism in Prior Analytics I 1 (24b18-22).
} 
inference $A a B, A o C \vdash_{s y l} B o C^{4}$, called 'Baroco', and an example of proof is the reductio ad impossibile in Prior Analytics I 5 that shows that Baroco is syllogistic (27a36-b1).

Aristotle uses three kinds of proof to show that inferences are syllogistic: deictic

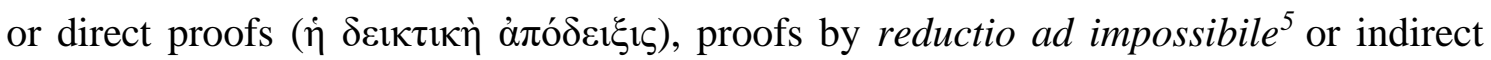

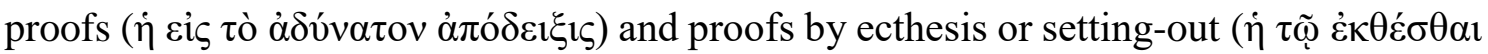
$\dot{\alpha} \pi$ ó $\delta \varepsilon \xi 1 \varsigma)$. Deictic proofs are the ones in which conversion is used, proofs by reductio ad impossibile make use of a hypothesis and in proofs by ecthesis a general proposition is proved by means of a singular one.

In this paper, I intend to characterize proofs by reductio ad impossibile in Aristotle's Prior Analytics, especially regarding what kinds of impossibility are suitable for such proofs to be carried out. The paper is divided in four sections. In the first section, some passages in which Aristotle describes reductio ad impossibile are analysed. In the second, scholars' accounts of what kinds of impossibility are suitable for such proofs, divided in traditional and alternative interpretations, are examined. In the third, textual evidence against the traditional interpretation is presented. Finally, in the fourth section, a definition of proof by reductio ad impossibile deemed to be in accordance with Aristotle's uses of it in the Prior Analytics is given and the alternative interpretation is argued for.

1 - Aristotle’s Statements on Reductio ad Impossibile

Let us start by examining passages in which Aristotle states what a proof by reductio ad impossibile is. He offers partial descriptions in various passages in the Prior Analytics. However, in Prior Analytics I 23 there is a passage in which he is fairly clear about it:

But it will be clear through these next considerations that this holds for deductions which lead into an impossibility as well. For all those which come to a conclusion through an impossibility

\footnotetext{
${ }^{4}$ The notation used for representing Aristotle's syllogistic is the standard one. Capital Roman letters stand for predicate variables, small Roman letters stand for a quantity and quality relation between predicates (" $a$ " stands for universal affirmative predication and so on) (For further explanation, see Striker 2009, p. 67). Let " $\vdash_{s y l}$ " stand for "syllogistic entailment". It should not be taken for granted that syllogistic entailment is the same as classical entailment, requiring only validity (see note 3 ).

${ }^{5}$ Proofs by reductio ad impossibile have been given many names throughout the history of philosophy: reductio ad impossibile, ad absurdum, per impossibile, indirect proof, etc. I will be mainly using 'proof by reductio ad impossibile' in this paper.
} 
deduce the falsehood, but prove the original thing from an assumption when something impossible results when its contradiction is supposed [...]. For this is what deducing through an impossibility was: proving something impossible by means of the initial assumption. ${ }^{6}(\operatorname{APr}$ I 23 , $41 \mathrm{a} 22-32)^{7}$

Aristotle's goal in this passage does not seem to be (only) to characterize proofs by reductio ad impossibile, but to show how they differ from direct proofs. While in the

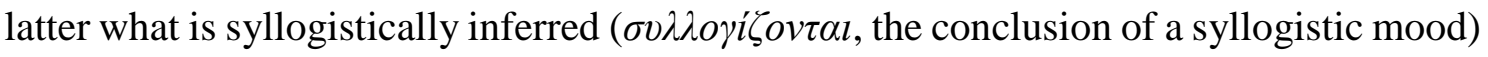
and what is proved ( $\delta \varepsilon \imath \kappa v v o v \sigma l v$, the conclusion of the proof) is the same, that clearly is not the case with proofs by reductio ad impossibile. According to the philosopher in the quoted passage, in these proofs, what is syllogistically inferred is a falsehood and what is proved is what was to be proved from the beginning. Given that what is proved, i.e., the conclusion of the proof, is true if the premises are true and what is syllogistically inferred, i.e., the conclusion of a syllogistic mood, is false, they cannot be the same proposition.

Nonetheless, by presenting this distinction, Aristotle describes the procedure of proofs by reductio ad impossibile fairly clearly. Summing up the passage, the proof consists in taking the contradictory of the proposition intended to be the conclusion of the proof as a hypothesis, syllogistically inferring something false or impossible from that hypothesis and thus prove that the intended proposition syllogistically follows from the premises because its contradictory following from them leads into an impossibility. Aristotle has left out only two points in the quoted passage: initially stating the premises of the syllogistic mood intended to be proved and stating that the premises for inferring (in a previously proved syllogistic mood) the impossibility must be the hypothesis (the contradictory of the intended conclusion) and one of the premises initially stated.

Therefore, combining these two remarks and what has been stated in the passage quoted above, a more detailed account of proofs by reductio ad impossibile can be given: first, the premises of the mood supposed to be proved are laid down. Second, the contradictory of the intended conclusion is assumed as a hypothesis. Third, the hypothesis and one of the premises from the first step are used for an inference in a previously proved

\footnotetext{
${ }^{6}$ The quoted passages of Aristotle's Prior Analytics and Topics used in this paper are from Smith's translations and the corresponding Greek text is from Ross' edition. Ackrill's translation was used for On Interpretation passages and the corresponding Greek text is from MinioPaluello's critical edition.

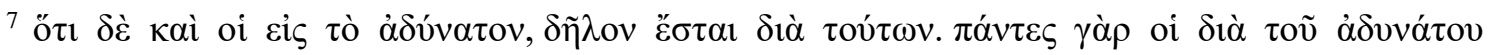

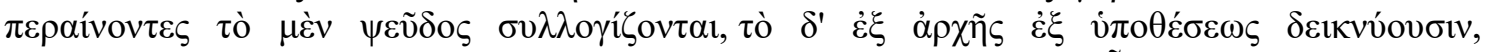

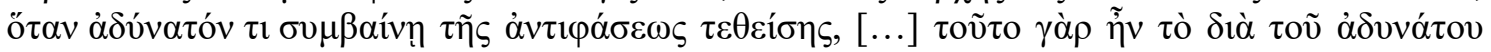

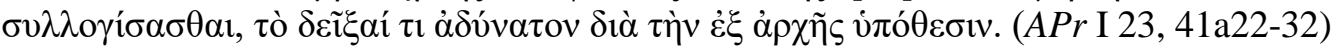


syllogistic mood. Next, the inconsistency between the conclusion inferred in the previous step and the other premise of the first step is stated, what makes holding the two of them an impossibility. Finally, since an impossibility follows from the assumed hypothesis, it is proved to be false and its contradictory, the intended conclusion, to be true (given the truth of the premises). Accordingly, it is proved that a certain conclusion follows from the premises laid down, which shows that these premises implying that conclusion constitutes a syllogistic mood. The structure of the proof can be written in the following way:

1 Premise $1(P 1)$.

2 Premise $2(P 2)$.

3 Hypothesis $($ Hyp $)$.

$\vdots \quad$ Repetition of 1 or 2 for the syllogistic inference (if required).

$n \quad$ Conclusion of a syllogistic inference whose premises are 1 and 3 or 2 and 3 (in any order).

$n+1$ Stating that $n$ and 1 or $n$ and 2 are inconsistent.

$n+2$ Conclusion of the contradictory of 3 , which is taken to be true because 3 is taken to be false due to $n+1$.

The omitted passage in the text quoted above (41a22-32) is an example of a proof by reductio ad impossibile, which is useful to show what its procedure is:

<proving,> for example, that the diagonal is incommensurable because if it is put as commensurable, then odd numbers become equal to even ones. It deduces that odd numbers become equal to even ones, then, but it proves the diagonal to be incommensurable from an assumption since a falsehood results by means of its contradiction. $(\operatorname{APr} \mathrm{I} 23,41 \mathrm{a} 26-30)^{8}$

The example shows that reductio ad impossibile is not a procedure created by Aristotle. Instead, the philosopher is using in his syllogistic a method of proof similar to one used elsewhere, judging from his example, in geometry ${ }^{9}$. Aristotle's example of proof by reductio ad impossibile is a proof of the incommensurability of the diagonal of a square with its sides. In this proof, there are no explicit premises from which the intended conclusion is supposed to follow. The intended conclusion is the diagonal is incommensurable' (short for 'the diagonal of a square is incommensurable with its sides'). Therefore, its contradictory ('the diagonal is not incommensurable' and therefore) 'the

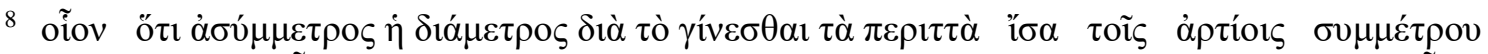

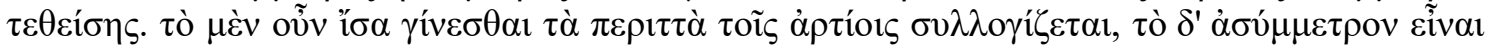

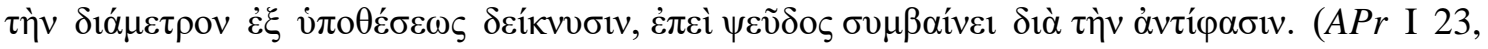
41a26-30)

${ }^{9}$ According to scholars, proofs by reductio ad impossibile were commonly used in Greek mathematics (Smith 1989, p. 115; Striker 2009, p. 70). 
diagonal is commensurable' is assumed as a hypothesis. From the hypothesis, somehow the proposition 'odd numbers are equal to even numbers' is inferred, which is taken to be evidently false. As the hypothesis entails falsehood, its contradictory 'the diagonal is incommensurable' must be true.

In Prior Analytics I 23 (41a22-32), Aristotle states that it is the contradictory of the intended conclusion that must be assumed as a hypothesis. The same point is repeated in several other passages. An example is in chapter 11 of book II: "A deduction through an impossibility is proved when the contradictory of the conclusion is put as a premise and one of the premises < of the deduction> is taken in addition [...]" (APr II 11, 61a18$21)^{10}$. Another is in chapter 14 of book II, where Aristotle says that a proof by reductio ad impossibile "takes one of these premises and, as other premise, the contradictory of the conclusion" ( $A P r$ II 14, 62b33-35) ${ }^{11}$. Considering only these statements, it is not evident why it is the contradictory of the intended conclusion that must be assumed as a hypothesis, and not any other opposite of the intended conclusion.

In Prior Analytics II 11 (62a11-19), Aristotle recognises at least one other opposite of the intended conclusion as a candidate to be the hypothesis of a proof by reductio ad impossibile, namely, the contrary of the conclusion. However, Aristotle resolutely refuses the contrary of the intended conclusion as a suitable hypothesis. Before examining his reasons for doing so, it is useful to go back to On Interpretation and review what contradiction and contrariety are. In On Interpretation 7, Aristotle states: "I call an affirmation and a negation contradictory opposites when what one signifies universally the other signifies not universally, e.g., 'every man is white' and 'not every man is white', 'no man is white' and 'some man is white'." (DI 7, 17b16-20) ${ }^{12}$. Regarding the truthvalue of contradictory propositions, Aristotle points out that one must be true and the other must be false: "Of contradictory statements about a universal taken universally it is

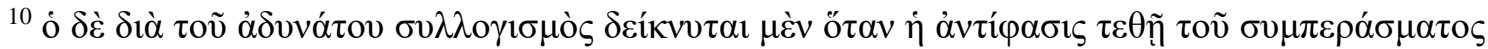

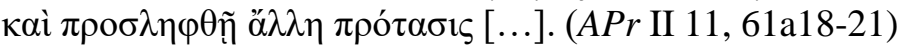

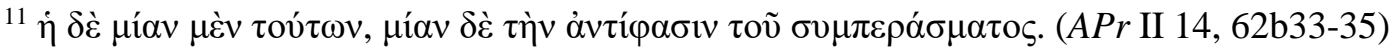
12

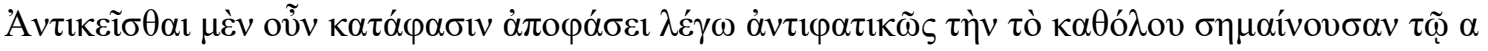

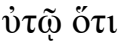

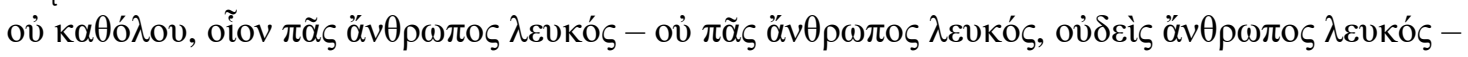

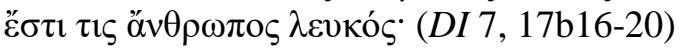


necessary for one or the other to be true or false." $(D I 7,17 \mathrm{~b} 26-27)^{13}$. Concerning contrary propositions, in On Interpretation 7 Aristotle writes: "But I call the universal affirmation and the universal negation contrary opposites, e.g. 'every man is just' and 'no man is just'. So these cannot be true together" $(D I 7,17 \mathrm{~b} 20-23)^{14}$. Thus, summing up the information obtained from $O n$ Interpretation 7, $A a B$ (universal affirmation) and $A o B$ (particular negation) as well as $A e B$ (universal negation) and $A i B$ (particular affirmation) are contradictory propositions. Of these pairs, one proposition must be true and the other false. On the other hand, $A a B$ (universal affirmation) and $A e B$ (universal negation) are contrary propositions. These cannot be both true, which leaves as possibilities that one of them be true and the other false or that they be both false.

Having stated what contradictory and contrary propositions are, let us examine Prior Analytics II 11 (62a11-19). In chapter 11 of book II, Aristotle explains why it is the contradictory of the intended conclusion that must be assumed as a hypothesis and why it cannot be its contrary:

It is evident, then, that it is the opposite, not the contrary, which must be assumed in all of the deductions. For in this way there will be a necessary result, and also the claim will be accepted. For if either the assertion or the denial is true of everything, then when it has been proved that the denial is not true, it is necessary for the affirmation to be true. Moreover, if someone does not put the affirmation to be true, then it is accepted to claim the denial. To claim the contrary, however, is not suitable in either way (for neither is it necessary for 'belongs to every' to be true if 'belongs to none' is false, nor is it accepted that if the one is false then the other is true). ( $\operatorname{Pr}$ II 11, 62a11$19)^{15}$

Proofs by reductio ad impossibile have the following proof strategy: proving the intended conclusion by proving that the hypothesis is false because it leads into an impossibility. In the quoted text, Aristotle gives two reasons why the hypothesis must be the contradictory and not the contrary of the intended conclusion. He describes such reasons in the following way: "For in this way there will be a necessary result, and also

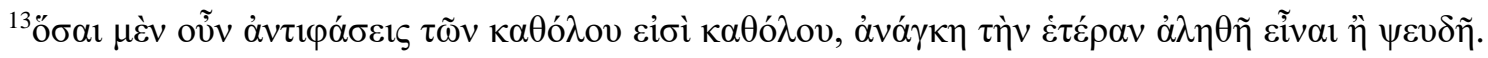
$(D I 7,17 \mathrm{~b} 26-27)$

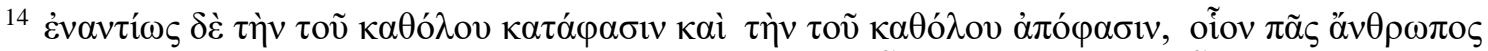

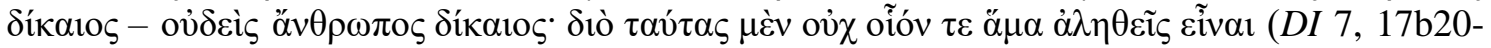
23)

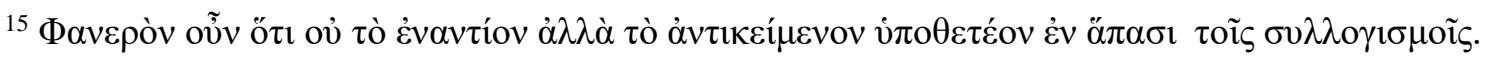

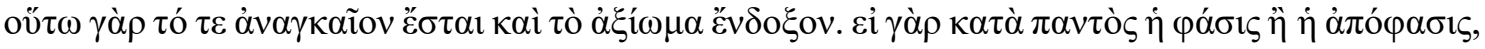

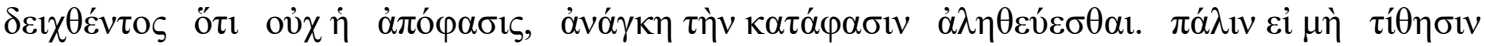

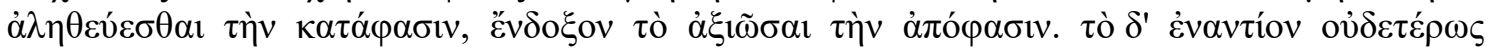

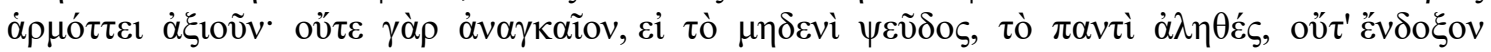

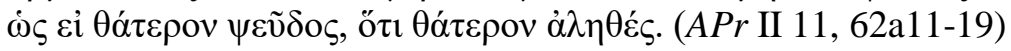


the claim will be accepted" (APr II 11, 62a12-13) ${ }^{16}$. The first reason, described as a 'necessary result', I will call 'logical reason'. It seems to be related to how truth-values are distributed in different pairs of opposite propositions. The second reason, described as 'the claim will be accepted', I will call 'dialectical reason'. This one seems to be related to what is convincing in a dialectical debate.

Starting with the logical reason, as it has been stated, proofs by reductio ad impossibile prove the intended conclusion by proving that the hypothesis is false because it leads into an impossibility. Moreover, according to On Interpretation 7, of contradictory propositions, it is necessary that one be true and the other be false (17b2627). Therefore, if the hypothesis of a reductio ad impossibile is the contradictory of the intended conclusion, by proving that the hypothesis is false, one has also proved that its contradictory is true, for if one proposition is false, its contradictory is true. Thus, one has obtained the intended conclusion. Aristotle's logical reason in the quoted passage of Prior Analytics II 11 for refusing contrary pairs of propositions for playing the roles of hypothesis and intended conclusion is that the proof strategy that works with contradictory propositions does not work with contrary ones. According to On Interpretation 7, contrary propositions cannot be both true simultaneously (17b20-23). Accordingly, if one proposition is true, then its contrary is false. However, nothing prevents both of them from being false. Consequently, by using contrary propositions for the roles mentioned, proofs by reductio ad impossibile cannot be carried out in the same way as before. For, if the hypothesis is the contrary of the intended conclusion, then proving that the hypothesis is false because it leads into an impossibility does not prove that its contrary is true nor false, because all that is necessary regarding the truth-values of contrary propositions is that they not be both true.

In Prior Analytics II 11 (62a11-19), Aristotle also gives a second reason for taking the contradictory of the intended conclusion as a hypothesis instead of its contrary, namely, the dialectical reason. The connection of this claim in Prior Analytics II 11 to Aristotle's dialectic is made clear by his use of the term ' $E$ 'v $\delta o \xi o v$ ' ('accepted') ${ }^{17}$. In

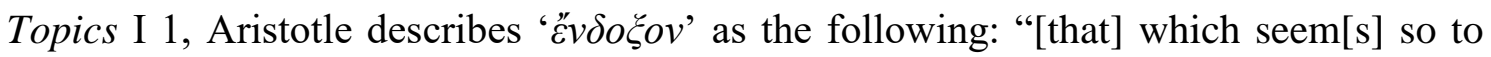
everyone, or to most people, or to the wise - to all of them, or to most, or to the most

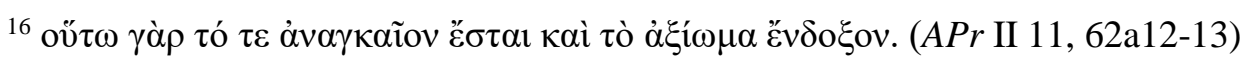

${ }^{17}$ Smith (1989, p. 200) points out the relation between the use of $\check{\varepsilon} v \delta o \xi o v$ in this passage and in Aristotle's Topics.
} 
famous and esteemed." (Top I 1, 100b21-23) ${ }^{18}$. In the passage of the Prior Analytics under discussion, Aristotle argues that if the hypothesis is proved to be false, then it is acceptable to claim that its contradictory is true. Nonetheless, the same is not the case if the hypothesis is the contrary of the intended conclusion. Aristotle argues that in this case, if the hypothesis is proved to be false, it is not acceptable (to people or to most or some of them, according to the specifications in Topics I 1, 100b21-23) to infer that its contrary is true.

Regarding a last aspect of proofs by reductio ad impossibile, namely, the impossibility that the hypothesis is supposed to entail, Aristotle does not explain what it

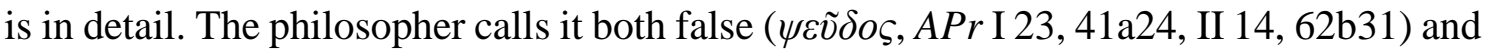

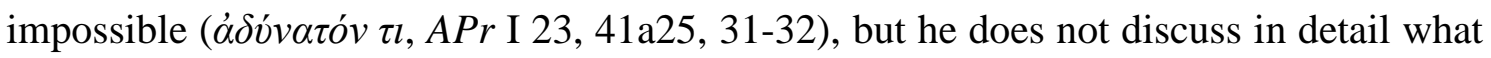
kinds of impossibility or falsehood he is referring to. In the next section, some scholars' interpretations of proofs by reductio ad impossibile will be presented, with special interest on their accounts of what this impossibility is.

\section{2 - Scholars’ Accounts of Reductio ad Impossibile}

The accounts of scholars who try to explain what Aristotle means by 'impossibility' in proofs by reductio ad impossibile in the Prior Analytics can be divided in two groups. The first group suggests it is a contradiction, i.e., the truth of two contradictory propositions. The second group suggests it is either a contrariety, i.e., the truth of two contrary propositions, or a contradiction. Some of the texts of scholars included in each of these groups will be examined in more detail in what follows, as paradigms of the interpretations of each group.

The first group of scholars take the impossibility in proofs by reductio ad impossibile to be a contradiction, i.e., the truth of two contradictory propositions. Therefore, in their account, the syllogism in a reductio ad impossibile must yield a proposition that holds a relation of contradiction to one of the premises. This interpretation is called by Patzig 'the traditional interpretation': "the 'impossible' to which reduction, on the traditional interpretation, leads, is meant to be [...], not a simple falsehood, but a contradiction between the second premiss of the original syllogism and

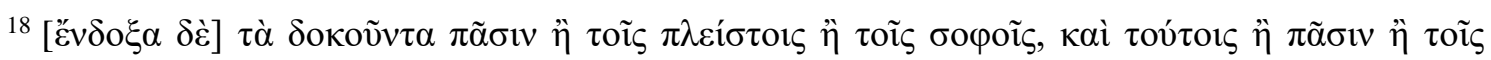

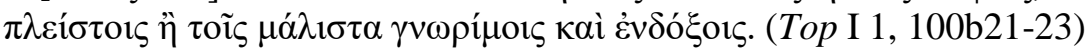


the proposition which, as described, is yielded by Barbara." (1968, p. 148). In this passage, Patzig is making a point about the traditional interpretation of the proof of Baroco in Prior Analytics I 5. However, this point can be generalized to provide an accurate description of the interpretation of the first group or 'traditional interpretation' of proofs by reductio ad impossibile. Thus, generalizing Patzig's statement, for the traditional interpretation, the impossibility to which reduction leads is meant to be not a simple falsehood, but a contradiction between a premise of the original syllogism and the proposition that is yielded by the syllogistic mood used in the proof. The group of scholars who subscribe to this traditional view includes Günther Patzig, John Corcoran, Timothy Smiley, Gisela Striker, Paolo Crivelli, Mateus Ferreira, Jan von Plato and Roy Dyckhoff. Their interpretations of the proofs under discussion will be analysed in what follows.

Günther Patzig, in his Aristotle's Theory of the Syllogism, expresses reductio ad impossibile through the propositional law "If from $p$ and the negation of $r$, not- $q$ follows, then $r$ follows from $(p$ and $q)$. In symbols: $(1)[(p \& \sim r) \rightarrow \sim q] \rightarrow[(p \& q) \rightarrow r]$ " (Patzig 1968 , p. 151). In this schema, ' $\sim q$ ' is the impossibility entailed by the hypothesis ' $\sim r$ '. ' $\sim q$ ' is an impossibility because it is the negation of the premise ' $q$ '. Given that this premise is assumed to be true, denying it is an impossibility, for it is contradictory to both affirm and deny $q$. Impossibility is expressed by Patzig in terms of contradiction, for, given the propositional law he chose to express proofs by reductio ad impossibile, the only kind of impossibility possible for these proofs is contradiction, since 'impossibility' is expressed in terms of affirming and denying the same proposition, i.e., $q$ and $\sim q$.

John Corcoran, in his Aristotle's Natural Deduction System, as well as in his Completeness of an Ancient Logic and A Mathematical Model of Aristotle's Syllogistic, gives the following definition of indirect deduction:

An indirect deduction in $D$ of $\mathrm{c}$ from $P$ is a finite list of sentences ending in a contradictory pair, beginning with a list of all or some of the sentences in $P$ followed by the contradictory of $\mathrm{c}$, and such that each subsequent additional line (after the contradictory of c) is either (a) a repetition of a previous line, (b) a D-conversion of a previous line or (c) a D-inference from two previous lines. (Corcoran 1973, p. 206; 1974, pp. 109-10; similar version in 1972, pp. 697-8)

The author explains the definition in ordinary language in 1972 as below:

An indirect deduction, on the other hand, does not contain its conclusion but rather it is, in effect, a direct deduction containing the contradictory of the conclusion as an added assumption and having a pair of contradictories for its last two lines. For Aristotle, an indirect proof of a 
conclusion from premises was obtained by deducing contradictory sentences from the premises together with the contradictory of the conclusion [...]. (Corcoran 1972, p. 697)

In addition, in 1973 and 1974, he gives a similar explanation:

In constructing an indirect deduction of a conclusion from premises one adds to the premises, as an additional hypothesis, the contradictory of the conclusion; then one interpolates new sentences as above until both of a pair of contradictory sentences have been reached. (Corcoran 1973, p. 205; 1974, p. 109)

A similar point is made in the 'reductio law', the semantic counterpart of the presented syntactic definition of indirect deduction: "Reductio Law: $(\mathrm{R}) P \vDash d$ if $P+$ $C(d) \vDash s$ and $P+C(d) \vDash C(s)$ " (Corcoran 1974, p. 106; similar version in 1972, p. 687), which he explains as "[t]he reductio law says that for $d$ to follow from $P$ it is sufficient that $P$ and the contradiction of $d$ together imply both a sentence $\mathrm{s}$ and its contradictory $C(s)$." (Corcoran 1974, p. 106). The same view is kept in later writings, such as his 2009 Aristotle's Demonstrative Logic:

The picture for an indirect deduction, or reductio-ad-impossibile, resembles but is significantly different from that for a direct deduction. Indirect demonstrations are called proofs by contradiction. In such a deduction, after the premises have been assumed and the conclusion has been set as a goal, the contradictory opposite of the conclusion is assumed as an auxiliary premise. Then, a series of intermediate conclusions are deduced until one is reached which oppositely contradicts a previous proposition. (Corcoran 2009, pp. 9-10)

In all the above passages, Corcoran clearly exposes his interpretation according to which a proof by reductio ad impossibile requires a pair of contradictory sentences to be entailed by the hypothesis and the initial premises for the proof to be carried out.

Timothy Smiley, in his What Is a Syllogism?, ascribes the form " $P$, suppose not $R$, then not $Q$, so $R$ " $(1973$, p. 136) to proofs by reductio ad impossibile and defines them as:

DEFINITION 1. (i) $\langle Q\rangle$ is a deduction of $Q$ from itself. (ii) If, for each $i,\left\langle\cdots P_{i}\right\rangle$ is a deduction of $P_{i}$, from $X_{i}$, and if $Q$ follows from $P_{1}, \ldots, P_{n}$ by a rule of inference, then $\left.<\cdots P_{1}, \ldots, \ldots P_{n}, Q\right\rangle$ is a deduction of $Q$ from $X_{1}, \ldots, X_{n}$. (iii) If $\langle\cdots P\rangle$ is a deduction of $P$ from $X_{1}, \bar{Q}$, and $\langle\cdots \bar{P}\rangle$ is a deduction of $\bar{P}$ from $X_{2}$, then $\langle\cdots P, \ldots \bar{P}, Q\rangle$ is a deduction of $Q$ from $X_{1}, X_{2}$. [...] The third clause is intended to accommodate reductio ad impossibile arguments. (Smiley 1973, pp. 141-2)

Smiley, as the authors above, defines reductio ad impossibile in propositional language. The impossibility in the proof is represented by $\langle\cdots P, \ldots \bar{P}\rangle$, a propositional expression for contradiction. When setting out the system that is supposed to include 
proofs by reductio ad impossibile in page 141, Smiley does not even define contrariety, but only contradiction. These evidences make clear that, according to Smiley's interpretation, contradiction alone can be the kind of impossibility entailed by the hypothesis for proofs by reductio ad impossibile to be carried out.

Gisela Striker, in the introduction of her translation and commentary on book I of the Prior Analytics, provides the following description of proofs by reductio ad impossibile:

Indirect proofs were well known from mathematics, and this may explain why Aristotle never explicitly formulates a corresponding rule. It might be stated as follows:

If an assumption used in a deduction leads to a contradiction, then the assumption is false and its contradictory must be true.

The typical case of a reductio-proof in chapters 5 and 6 is very simple: given the two premisses of a syllogistic mood, one adds the contradictory of the expected conclusion as a hypothesis and then derives the contradictory of one of the premisses from the hypothesis together with the other premiss. Obviously, the two premisses are supposed to be true, so that the contradiction can only be due to the hypothesis. (Striker 2009, p. 70)

Striker too thinks that the hypothesis must lead to a contradiction for a proof by reductio ad impossibile to be carried out. The contradiction holds between the conclusion of a syllogism that has the hypothesis and one of the premises of the syllogistic mood as its premises and the other premise of the syllogistic mood.

Paolo Crivelli, in his Aristotle's Logic, gives the following definition of reductio ad impossibile: "PI [per impossibile] If from certain premises a certain conclusion is inferred, then any contradictory of any of those premises may be inferred from the result of replacing that premise with any contradictory or contrary of that conclusion." (Crivelli 2012). In Prior Analytics II 11 (62a11-19), Aristotle shows that not the contrary but only the contradictory of the conclusion can be assumed as a hypothesis (the premise replaced, in Crivelli's definition), as it has been discussed in section 1. By stating in his definition that the contradictory of one of the premises is what is attained in a proof by reductio ad impossibile, Crivelli assumes the thesis endorsed by the first group.

Mateus Ferreira, in section 6 of his $O$ que são silogismos perfeitos?, presents a natural deduction system for Aristotle's syllogistic. Among the rules of the system, Ferreira introduces one called 'rule for indirect proof', which is the following: "RA (Reduction to the absurd). $\alpha$; if $\neg \beta$, then $\neg \alpha$; then, $\beta . " 19$ (Ferreira 2013, p. 213, my translation). According to RA, the impossibility that the hypothesis must entail for the

19 'RA (redução ao absurdo). $\alpha$; se $\neg \beta$, então $\neg \alpha$; então, $\beta$.' (Ferreira 2013, p. 213) 
proof to be carried out is a contradiction, composed of a premise and a proposition obtained from the hypothesis. As it has been said above, that is the traditional interpretation.

Jan von Plato, in his The Great Formal Machinery Works: Theories of Deduction and Computation at the Origins of the Digital Age, as well in his Elements of Logical Reasoning and Aristotle's deductive logic: A proof-theoretical study, gives the following description of proofs by reductio ad impossibile:

(B) THE PRINCIPLE OF INDIRECT PROOF. The two pairs Every $A$ is $B$, Some $A$ is not- $B$ and No $A$ is $B$, Some $A$ is $B$ form between themselves contradictory opposites. Furthermore, because from $N o A$ is $B$ the weaker Some $A$ is not-B follows, also Every $A$ is $B$ and No $A$ is $B$ together lead to a contradictory pair. We indicate the contradictory opposite of a proposition $P$ by the orthogonality symbol, $P^{\perp}$. (Note that $P^{\perp \perp}$ is identical to $P$.) In general, if an assumption $P$ has led to contradictory consequences $Q$ and $Q^{\perp}, P^{\perp}$ can be concluded and the assumption $P$ closed. [...] A rule of indirect proof in which the premisses of RAA [reductio ad absurdum] are Every $A$ is $B$ and its contrary No $A$ is $B$ can be derived from the second of the following conversion rules $\left[\frac{\text { Every } A \text { is } B}{\text { Some } B \text { is } A}\right]$. (von Plato 2017, pp. 9-10, a similar version in 2013, pp. 222-3 and 2016, pp. 3289)

The most relevant point for this discussion in von Plato's description is that he reduces contrariety to contradiction. Given that e-propositions imply o-propositions, then a-propositions and e-propositions are incompatible because a-propositions and opropositions are incompatible. Moreover, given that, from conversion, a-propositions imply i-propositions, then the incompatibility of a-propositions and e-propositions can be reduced to the incompatibility between e-proposition and i-propositions. Thus, the incompatibility between a-propositions and e-propositions is reduced to the one between a-propositions and o-propositions or the one between e-proposition and i-propositions. Therefore, stricto sensu, von Plato's conception of proofs by reductio ad impossibile admits only contradictions as the impossibility derived in these proofs, for contrariety is reduced to contradiction.

Roy Dyckhoff, in the syllogistic system he defines in his Indirect Proof and Inversion of syllogisms, suggests the following rule to play the role of indirect proofs (IP):

IP: If we have deduced $B$ from $A^{*}$ and also have deduced $B^{*}$, then we may combine the two deductions, remove (i.e., discharge) the single assumption of $A^{*}$ and thus form a deduction of $A$ (from the multiset sum of the two multisets of undischarged assumptions). (Dyckhoff 2019, p. 198)

In Dyckhoff's notation, ' $A$ *' stands for 'the contradictory of $A$ '. Therefore, in his interpretation, proofs by reductio ad impossibile include only cases in which 
contradictory propositions ( $B$ and $B *$ in the quoted passage) are entailed by the hypothesis $\left(A^{*}\right)$, which is the traditional interpretation.

The second group of scholars presents an alternative interpretation of the impossibility in proofs by reductio ad impossibile, taking it to be either a contrariety, i.e., the truth of two contrary propositions, or a contradiction. Thus, according to them, the syllogism in a reductio ad impossibile yields a proposition that holds a relation of either contrariety or contradiction to one of the premises. This group of scholars includes William of Ockham, Robin Smith, Marko Malink and Stephen Read. Their accounts will be exposed in what follows.

William of Ockham, in his exposition of Aristotle's Prior Analytics in Summa Logicae III-1, gives the following account of proofs by reductio ad impossibile in the second figure:

Therefore every syllogism in the second figure is reduced to the syllogisms in the first figure, namely [to those] in the first two moods, always asserting from the major as the prior [proposition] and the contrary or the contradictory of the conclusion [as the posterior], inferring the contrary or the contradictory of the minor, always in virtue of the rule 'if [a proposition] incompatible with the conclusion does not stand with the antecedent, then the first consequence is sound. ${ }^{20}$ (Ockham, Summa Logicae III-1 11, 50-55, my translation $)^{21}$

In this passage, Ockham refers to the use of proofs by reductio ad impossibile to reduce the syllogistic moods in the second figure to those in the first one. According to him, in such proofs either the contrary or the contradictory of the minor premise is attained from the major premise and the contrary or the contradictory of the intended conclusion. Ockham allows reductio proofs to take either the contrary or the contradictory of the conclusion as a hypothesis, which Aristotle clearly argues against in Prior Analytics II 11, as it has been discussed in section 1. On the other hand, allowing proofs by reductio ad impossibile to have either contrariety or contradiction as the impossibility entailed by the hypothesis includes Ockham in the second group of scholars announced above.

Robin Smith, in the introduction of his translation and commentary on the Prior Analytics, presents the following structure for proofs by reductio ad impossibile:

\footnotetext{
${ }^{20}$ The Latin text for this passage of William of Ockham's Summa Logicae is from Boehner's (et al.) edition.

${ }^{21}$ Sic igitur omnis syllogismus secundae figurae reducitur in syllogismos primae figurae, scilicet in duos primos modos, arguendo semper ex maiore qua prius et contraria vel contradictoria conclusionis, inferendo contrariam vel contradictoriam minoris, semper virtute istius regulae 'repugnans conclusionis non stat cum antecedente, igitur prima consequentia bona'. (Ockham, Summa Logicae III-1 11, 50-55)
} 
A deduction through impossibility has the following structure (for 'the contradictory of $s^{\prime}$ I write 'Cont(s))':

Premise 1

Premise 2

Cont(Conclusion)

Step 1

$\cdots$

Step $n=\operatorname{Cont}($ Premise 1) or Cont(Premise 2) (Smith 1989, p. XXI)

According to Smith, the last step of the proof consists in inferring from the hypothesis and one of the premises the contradictory either of the first or of the second premise. Therefore, the impossibility to which the hypothesis leads is a contradiction between the first premise or the second premise and the conclusion of the syllogism whose premises are either the first premise or the second premise and the hypothesis. This account alone would include Smith in the group of scholars who adopt the traditional interpretation. However, in his later writing Logic, Smith seems to change his account:

Sometimes, Aristotle must use another pattern of proof, namely completion through impossibility. $\mathrm{He}$ adds the denial of the desired conclusion to the premises and, from this and one of the original premises, deduces the contradictory [or contrary] of the other premise. This shows that the original premises and the denial of the conclusion cannot all be true; therefore, if the premises are true then the denial of the conclusion must be false [i.e. the conclusion must be true]. (Smith 1995, pp. 38-9)

Although the concession is made within brackets, Smith allows that the contradictory or the contrary of one of the premises be suitable as an impossibility for proofs by reductio ad impossibile, which includes him in the second group of scholars.

Marko Malink, in his Aristotle's Modal Syllogistic, gives the following description of reductio ad impossibile:

Aristotle does not explicitly formulate a rule for indirect deductions. It is, however, clear that indirect deductions involve a step of assuming for reductio the contradictory of the intended conclusion. Aristotle determines the contradictories of assertoric propositions as follows:

$A a x B$ is the contradictory of $A o x B$, and vice versa

$A \operatorname{ex} B$ is the contradictory of $\operatorname{Aix} B$, and vice versa

Moreover, in some of his indirect deductions, Aristotle avails himself of the following principle concerning the incompatibility of $a x-$ and $e x$ - propositions:

$A a x B$ is incompatible with $A \operatorname{ex} B$, and vice versa

Given these principles of contradictoriness and incompatibility, Aristotle's method of indirect deduction can be described as follows. First some premises are assumed. Then the contradictory of the intended conclusion is assumed for a reductio as an additional premise. Based on the resulting extended set of premises, we begin to construct a direct deduction. We try to go on until the direct deduction contains two propositions that are contradictory to or incompatible with each other. If successful, we have given an indirect deduction of the intended conclusion from the original premises. (Malink 2013, p. 31-2) 
Malink's account of reductio ad impossibile is more inclusive, if compared to the accounts of the scholars who endorse the traditional interpretation. He affirms that the impossibility that the premises together with the reductio hypothesis entail can be either a pair of contradictory or incompatible propositions. 'Incompatible' is not be the best term choice, for both contradictory and contrary propositions are incompatible. Based on his formulation of a principle to express incompatibility using a-propositions and epropositions, he probably means contrary propositions. Thus, his account of reductio ad impossibile is that the impossibility that the premises together with the reductio hypothesis entail can be either a pair of contradictory or contrary propositions, which is the alternative interpretation of proofs by reductio ad impossibile.

Stephen Read, in his Aristotle's Theory of the Assertoric Syllogism, gives the same account: "Note that the subproof in a reductio proof need only conclude in contraries (though often, as above, they are in fact contradictories). But the assumption for reductio must, of course, be the contradictory of the ultimate conclusion to be proved." ( $\operatorname{Read} 2017$, p. 10). In a short but accurate formulation, Read asserts that both contrary and contradictory propositions are suitable as what is entailed by the hypothesis and one of the premises in a proof by reductio ad impossibile, although in most cases it is contradictory propositions. That is, as stated above, the view of the second group.

What is the precise account of impossibility in proofs by reductio ad impossibile is clearly controversial, since some scholars allow only contradiction as suitable, whereas others allow both contradiction and contrariety. Scholars seem not to have discussed such controversy, nor do they argue for the definitions or descriptions of reductio ad impossibile they set forth, making it seem that the point in question is well established. However, as it has been shown in this section, there is disagreement between two positions, which I named traditional and alternative interpretations. The disagreement lies in what kinds of impossibility should be included in the definition of reductio ad impossibile as a suitable impossibility for such proofs to be carried out. More precisely, the disagreement lies in whether or not to include contrariety as an impossibility suitable for the purpose in question. In the next section, I will present some textual evidence in the Prior Analytics that proves the traditional interpretation to be too restricted and the alternative interpretation to be the appropriate one. 


\section{3 - Some Proofs by Reductio ad Impossibile in Prior Analytics I 5-7}

In this section, I will present Aristotle's proof that Baroco is a syllogistic mood using reductio ad impossibile in chapter 5 of book $\mathrm{I}$, which is the first proof by reductio ad impossibile presented in the Prior Analytics. This proof of Baroco is a paradigm of what most scholars consider a proof by reductio ad impossibile in the Prior Analytics to be. It should be noticed that the impossibility to which this proof leads is the truth of contradictory propositions. Following that, most scholars define proofs by reductio ad impossibile as requiring a contradiction as the impossibility led into by the hypothesis, as it has been shown to be the account of scholars who subscribe to the traditional interpretation in section two. Against those accounts, and in favour of the alternative interpretation, I will present two proofs by reductio ad impossibile in which the impossibility that follows from the hypothesis is not the truth two of contradictory propositions, but of two contrary ones.

Let us start with the paradigm of proofs by reductio ad impossibile. In Prior Analytics I 5, Aristotle proves that Baroco is a syllogistic mood using reductio ad impossibile:

Next, if $\mathrm{M}$ belongs to every $\mathrm{N}$ but does not belong to some $\mathrm{X}$, it is necessary for $\mathrm{N}$ not to belong to some $\mathrm{X}$. (For if it belongs to every $\mathrm{X}$ and $\mathrm{M}$ is also predicated of every $\mathrm{N}$, then it is necessary for $\mathrm{M}$ to belong to every $\mathrm{X}$ : but it was assumed not to belong to some.) $(\operatorname{APr} \mathrm{I} 5,27 \mathrm{a} 36-\mathrm{b} 1)^{22}$

For this proof, MaN and $M o X$ are assumed as premises and $\mathrm{NaX}$, the contradictory of the expected conclusion $N o X$, as a hypothesis. Then, $M a X$ is obtained by applying Barbara to the first premise, MaN, and to the hypothesis, $\mathrm{NaX}$. MaX, the obtained result, and $M o X$, the second premise, are contradictory propositions. Thus, the assumption of the truth of both constitutes an inconsistency in the proof. That entails that the assumed hypothesis is false. Therefore, its contradictory, NoX, must be true. The expected conclusion is attained and Baroco is proved to be a syllogistic mood. The proof can be represented as follows:

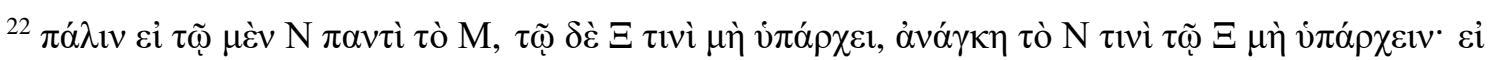

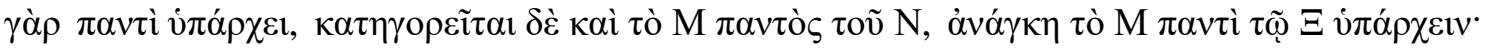

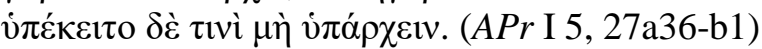




$\begin{array}{ccc} & \text { MaN } & \operatorname{Pr}^{23} \\ 1 & \text { MoX } & \operatorname{Pr} \\ 2 & \text { NaX } & H y p \\ 3 & \text { MaX } & \text { Bar, } 1,3 \\ 4 & \text { MaX -MoX } & I 2,2,4 \\ 5 & & 3,5 \\ 6 & \text { NoX } & \end{array}$

Let us now proceed to two proofs by reductio ad impossibile in which the impossibility entailed by the hypothesis is not contradiction, but contrariety. In Prior Analytics I 7, Aristotle gives a proof by reductio ad impossibile that Darapti is a syllogistic mood, which he had already proved by conversion and by exposition in chapter 6:

As, for instance, it is proved in the last figure that if both A and B belong to every C, then A will belong to some $\mathrm{B}$ : for if it belongs to none and $\mathrm{B}$ to every $\mathrm{C}$, then $\mathrm{A}$ will belong to no $\mathrm{C}$ : but it belonged to every C. $(A \operatorname{Pr} \text { I 7, 29a36-39) })^{24}$

For this proof, $A a C$ and $B a C$ are assumed as premises and $A e B$, the contradictory of the expected conclusion $A i B$, as a hypothesis. Then, $A e C$ is obtained by applying Celarent to the hypothesis, $A e B$, and to the second premise, $B a C$. $A a C$, the first premise, and $A e C$, the obtained conclusion, are contraries. Thus, the assumption of the truth of both constitutes an inconsistency in the proof. That entails that the assumed hypothesis is false. Therefore, its contradictory, $A i B$, must be true. The expected conclusion is attained and Darapti is proved to be a syllogistic mood. The proof can be represented as follows:

$\begin{array}{ccc}1 & A a C & P r \\ 2 & B a C & P r \\ 3 & A e B & H y p \\ 4 & B a C & R e p 25,2 \\ 5 & A e C & C e l, 3,4 \\ 6 & A a C-A e C & I 1,1,5 \\ 7 & A i B & 3,6\end{array}$

\footnotetext{
${ }^{23}$ In the proofs, let ' $\mathrm{Pr}$ ' stand for 'premise', 'Hyp' for 'hypothesis', 'Bar' for 'Barbara' and ' $I 2$ ' for 'contradiction'.

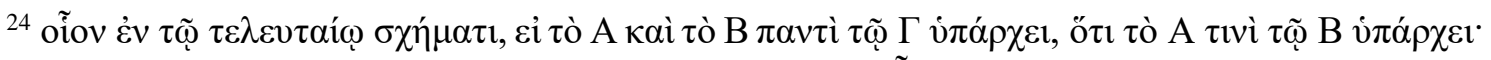

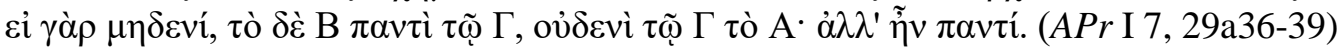

${ }^{25}$ In the proofs, let 'Rep' stand for 'repetition, 'Cel' for 'Celarent' and ' 11 ' for 'contrariety'.
} 
In Prior Analytics I 6, Aristotle states that Felapton can be proved to be a syllogistic mood by reductio ad impossibile. There he gives the proof by conversion, but not the one by reductio ad impossibile:

And if $\mathrm{R}$ belongs to every $\mathrm{S}$ but $\mathrm{P}$ to none, then there will be a deduction that $\mathrm{P}$ of necessity does not belong to some $\mathrm{R}$ (for the manner of demonstration is the same if premise RS is converted, and it could also be proved through an impossibility as in the previous cases). ( $A P r$ I 6, 28a26$30)^{26}$

Although Aristotle did not construct this proof himself, I will present it below. For a proof of Felapton in the first figure, $P e S$ and $R a S$ are assumed as premises and $P a R$, the contradictory of the expected conclusion $P o R$, as a hypothesis. Then, $P a S$ is obtained by applying Barbara to the hypothesis, PaR, and to the second premise, RaS. PeS, the first premise, and $P a S$, the obtained conclusion, are contrary propositions. Thus, the assumption of the truth of both constitutes an inconsistency in the proof. That entails that the assumed hypothesis is false. Therefore, its contradictory, PoR, must be true. The expected conclusion is attained and Felapton is proved to be a syllogistic mood. The proof can be represented as follows:

$\begin{array}{ccc}1 & P e S & P r \\ 2 & R a S & P r \\ 3 & P a R & H y p \\ 4 & R a S & \text { Rep }, 2 \\ 5 & P a S & \text { Bar, } 3,4 \\ 6 & P a S-P e S & I 1,1,5 \\ 7 & \text { PoR } & 3,6\end{array}$

The proofs by reductio ad impossibile given by Aristotle of Baroco in Prior Analytics I 5 and of Darapti in Prior Analytics I 7 have been presented and the one of Felapton in Prior Analytics I 6 not given by Aristotle has been constructed. It has been shown that the proofs by reductio ad impossibile that Darapti and Felapton are syllogistic moods have the truth of two contrary propositions as the impossibility entailed by the hypothesis. Therefore, these two cases are evidence for the acceptance of contrariety as a suitable kind of impossibility in proofs by reductio ad impossibile in the Prior Analytics,

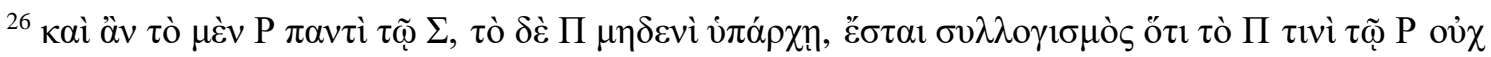

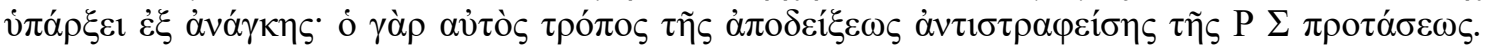

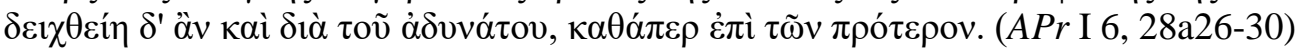


against the traditional interpretation of Aristotle's account of reductio ad impossibile and in accordance with the alternative one.

4 - A Definition of Proof by Reductio ad Impossibile

Let the following be a description of what a proof by reductio ad impossibile is according to the passages examined in section 1.

(I) Proof by reductio ad impossibile. A finite sequence of propositions is a proof by reductio ad impossibile in Aristotle's syllogistic if and only if every proposition in it is either (i) a premise, (ii) a hypothesis, (iii) the repetition of a premise, (iv) the conclusion of a syllogistic inference from (i) and (ii) or (ii) and (iii), (v) the statement of an inconsistency in (i) and (iv), or (vi) the statement of the contradictory of (ii) due to (v). ${ }^{27}$

The following is a schema of (I):

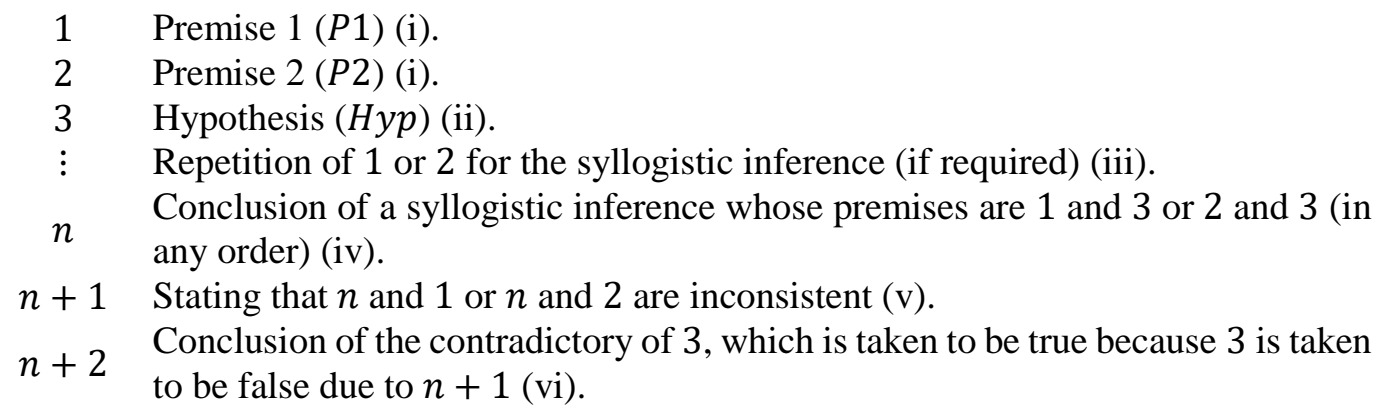

As it has been argued in section 1, Aristotle does not state what kinds of inconsistency are suitable for (v), for he describes such inconsistencies merely as impossible and false. This way, it falls to the reader of Aristotle's text to infer from the proofs given in the Prior Analytics what kinds of impossibility are suitable for proofs by reductio ad impossibile to be carried out.

As it has been argued in section 2, although such a difference is not stressed in the literature, there seems to be disagreement among scholars regarding what kinds of inconsistency are suitable for a proof by reductio ad impossibile. Some scholars define

\footnotetext{
${ }^{27}$ Let proposition be any string of symbols of the form $\alpha x \beta$ in which $a$ and $\beta$ are substituted for predicate terms and $x$ for $a$ (universal affirmation), $e$ (universal negation), $i$ (particular affirmation) or $o$ (particular negation). Regarding 'premise' and 'hypothesis', both are propositions, but the former is assumed to be true and the latter only conditionally taken to be true, in such a way that, if any inconsistencies come up, they are known to be due to the hypothesis and, therefore, the hypothesis is taken to be false. Concerning the syllogistic inferences admissible for step (iv), let them be any mood of inference previously proved to be syllogistic.
} 
reductio ad impossibile as a procedure that admits only contradictions as the inconsistency required for the proof to be carried out, which I named 'traditional interpretation', for this reading seems to be more common among scholars than its alternative version. Other scholars define reductio ad impossibile as a procedure that admits both contradictions and contrarieties as the inconsistency required for the proof to be carried out, which I named 'alternative interpretation'.

Thus, the preliminary description of reductio ad impossibile (I) presented is vague and can be read as either of the two following definitions:

(I.1) Proof by reductio ad contradictionem. A finite sequence of propositions is a proof by reductio ad impossibile in Aristotle's syllogistic if and only if every proposition in it is either (i) a premise, (ii) a hypothesis, (iii) the repetition of a premise, (iv) the conclusion of a syllogistic inference from (i) and (ii) or (ii) and (iii), (v) the statement of a contradiction in (i) and (iv), or (vi) the statement of the contradictory of (ii) due to (v).

The following is a schema of (I.1):

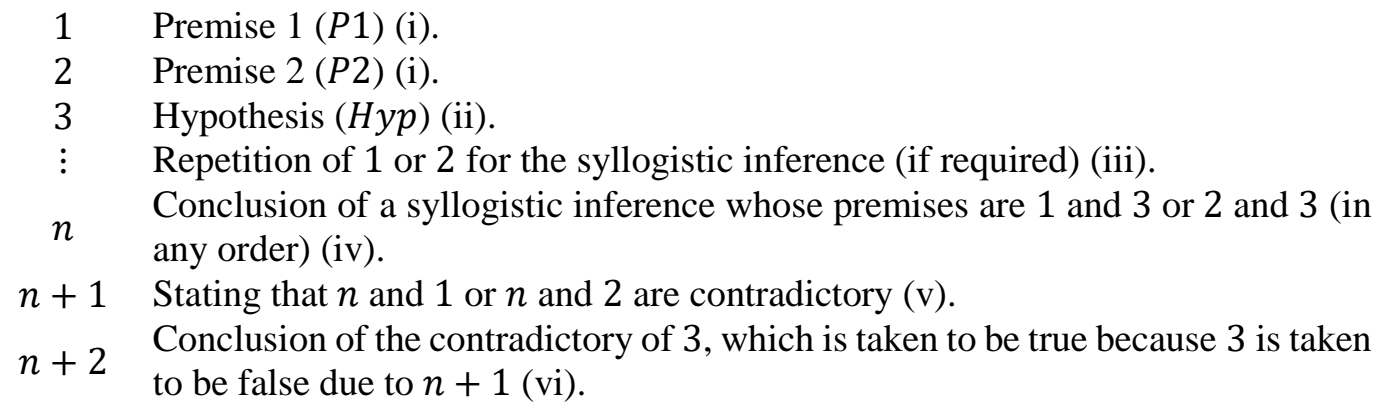

(I.2) Proof by reductio ad contradictionem vel contrarietatem. A finite sequence of propositions is a proof by reductio ad impossibile in Aristotle's syllogistic if and only if every proposition in it is either (i) a premise, (ii) a hypothesis, (iii) the repetition of a premise, (iv) the conclusion of a syllogistic inference from (i) and (ii) or (ii) and (iii), (v) the statement of a contradiction or a contrariety in (i) and (iv), or (vi) the statement of the contradictory of (ii) due to (v).

The following is a schema of (I.2):

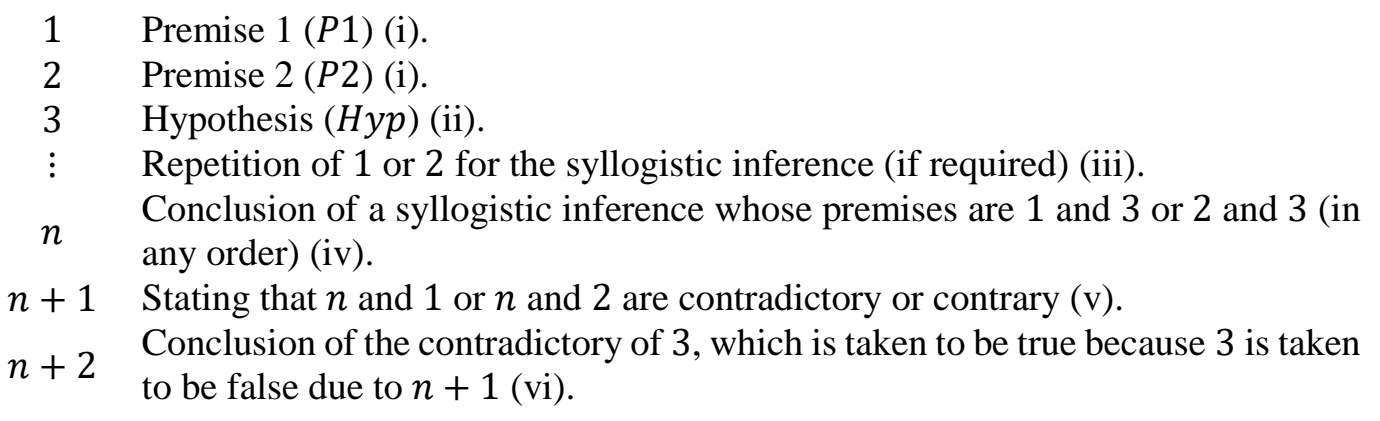

Reductio ad contradictionem (I.1) is what the traditional interpretation considers Aristotle's proofs by reductio ad impossibile to be, whereas reductio ad contradictionem vel contrarietatem (I.2) is how the alternative interpretation understands them. 
One might try to argue that the two interpretations are not different at all. It could be claimed that, although the traditional interpretation includes only contradiction as an acceptable impossibility in the definition of reductio ad impossibile, as contrarieties imply contradictions, the traditional interpretation would also indirectly accept contrarieties as suitable impossibilities for the proofs in question. Thus, the notion of reductio ad impossibile endorsed by the traditional interpretation would amount to the one endorsed by the alternative interpretation.

It should be noticed that contrariety implying contradiction lies on the supposition of existential import. It could be argued, in favour of the claim under discussion, that a contrariety, i.e., the relation between propositions $A a B$ and $A e B$, implies contradiction, i.e., either the relation between propositions $A a B$ and $A o B$ or $A e B$ and $A i B$. That implication requires subalternation: $A a B \vdash_{s y l} A i B$ and $A e B \vdash_{s y l} A o B$. However, subalternation presupposes existential import. Subalternation rules hold only if universal propositions have existential import, so particular propositions can be derived from them. Nonetheless, Aristotle's commitment to existential import is known to be a controversial matter ${ }^{28}$. This, of course, does not rule out contrariety implying contradiction, but does put it in question. Therefore, arguing that the definition of the traditional interpretation indirectly includes contrariety lies on controversial grounds.

Putting this controversial matter aside, there are further reasons for arguing that the two interpretations do not amount to the same understanding of reductio ad impossibile. First, every proof by reductio ad contradictionem (I.1) will have one step more than proofs by reductio ad contradictionem vel contrarietatem (I.2), for stating a contrariety as inconsistency is not enough for the former proofs, but one always has to explicitly derive a contradiction from it, whereas in the latter proofs that is not required.

Moreover, traditional and alternative interpreters clearly do not have the same definition of reductio ad impossibile (I). Reductio ad contradictionem (I.1) definitionally requires a contradiction for the reductio to be carried out. On the other hand, reductio ad contradictionem vel contrarietatem (I.2) definitionally requires either a contradiction or

\footnotetext{
${ }^{28}$ For some problems regarding the existential import supposition in Aristotle, see Smith (1989, p. xxv-xxvi) and Mignucci (2007). Of course, the first subalternation rule $\left(A a B \vdash_{s y l} A i B\right)$ can be obtained using the $a$-conversion $\left(A a B \vdash_{s y l} B i A\right)$ and the $i$-conversion $\left(A i B \vdash_{s y l} B i A\right)$ rules presented by Aristotle in Prior Analytics I 2. The second subalternation rule $\left(A e B \vdash_{s y l} A o B\right)$ might be obtainable in some other way. However, by using conversion rules instead of subalternation ones, one does not get rid of the existential import supposition, for conversion rules require existential import as well (Smith 1989, p. xxv-xxvi).
} 
a contrariety. Such different definitions of reductio ad impossibile are a result of interpreters having different notions of it. Traditional interpreters have a notion of reductio ad impossibile for which contradiction is necessary and sufficient, whereas contrariety is not necessary nor sufficient. On the other hand, alternative interpreters have a notion of reductio ad impossibile for which either contradiction or contrariety is necessary and sufficient. Thus, the requirements of the two definitions and the properties of the two notions are not the same.

All of the reasons listed above try to show that traditional and alternative interpretations are different through logical means. Exegetically, it is easier to show the point. In section 3, it has been shown that two of Aristotle's proofs by reductio ad impossibile in assertoric syllogistic require contrariety as an impossibility. One of them, the proof of Darapti in Prior Analytics I 7, was constructed by Aristotle himself. In this proof, he uses a contrariety to carry out the reductio without reducing contrariety to contradiction. Nonetheless, traditional interpretation requires that a contradiction appear in a proof by reductio ad impossibile. Therefore, according to their definitions, Aristotle's proof of Darapti in Prior Analytics I 7 would not be a well-constructed proof by reductio ad impossibile, for no contradiction appears in it. Thus, none of the definitions or descriptions of reductio ad impossibile presented by traditional interpreters in section 2 is a suitable reconstitution of Aristotle's definition or notion of proof by reductio ad impossibile, for none of them include the proof of Darapti, which Aristotle recognises as a proof by reductio ad impossibile. The definitions set forth by alternative interpreters, on the other hand, are suitable reconstitutions of Aristotle's notion, for they include both the proofs included by the traditional interpretation and the counterexample to it, namely, the proof of Darapti. This is enough to show that the interpretations are different and that one is exegetically adequate, whereas the other is not.

An aspect of the definitions of reductio ad impossibile in the traditional interpretation that is likely misleading is the use of propositional language, especially in the formulation of inconsistency. Many of them represent the impossibility in the proof in schemata such as ' $P$ and its negation' or ' $P$ and $\neg P$.' These formulations correspond to only one of Aristotle's kinds of inconsistency, namely contradiction. Aristotle's predicate language used for syllogistic contains at least one other kind inconsistency besides contradiction, namely, contrariety. It has been shown that contrariety is, alongside contradiction, an admissible kind of inconsistency for proofs by reductio ad impossibile. As definitions of proof by reductio ad impossibile in propositional language seem to lead 
into taking only contradiction as inconsistency, for contrariety is not expressible in it, predicate language seems to be more suitable for defining Aristotle's reductio ad impossibile and representing inconsistency in the Prior Analytics.

According to definitions (I.1) and (I.2), every reductio ad contradictionem is a reductio ad contradictionem vel contrarietatem, but the converse proposition does not hold universally, for although some reductio ad contradictionem vel contrarietatem are reductio ad contradictionem, some are not, e.g., the proofs of Darapti and Felapton. Therefore, the definition of reductio ad impossibile ascribed to Aristotle by the traditional interpretation, which I called 'proof by reductio ad contradictionem' (I.1), is not a suitable definition for Aristotle's procedure of reductio in the Prior Analytics, for there are (at least) two cases of reductio ad impossibile, recognized by Aristotle as such, which are not included by the definition mentioned. Therefore, I argue that Aristotle is not committed to (I.1), but instead to the definition of reductio ad impossibile ascribed to him by the alternative interpretation, which I called 'proof by reductio ad contradictionem vel contrarietatem' (I.2).

For proving the claim of this paper, that the traditional interpretation of reductio ad impossibile does not correspond to Aristotle's account of it, but that the alternative interpretation of it does, I have analysed only proofs in assertoric (i.e., non-modal) syllogistic in book I of the Prior Analytics. More evidence for the alternative interpretation point might be found elsewhere. However, one example of a proof by reductio ad impossibile recognized by Aristotle as so and not by the traditional interpretation is enough to refuse their definition, and I have presented two, of which at least one, the proof of Darapti, is uncontroversial.

Thus, some of Aristotle's passages on reductio ad impossibile have been analised, as have been scholars' accounts of it, which were divided in traditional and alternative interpretations. Then, textual evidence has been presented against the traditional interpretation and in favour of the alternative one. Finally, the definition of reductio ad impossibile of each interpretation has been presented and it has been summed up why the alternative interpretation is preferable to the traditional one.

Antônio Mesquita Neto Universidade Federal de Goiás 


\section{References}

Aristoteles. Aristotelis Categoriae et Liber de Interpretatione. Ed. L. Minio-Paluello. Oxford: Clarendon Press, 1949.

Aristoteles. Aristotelis Topica et Sophistici Elenchi. Ed. W. D. Ross. Oxford: Clarendon Press, 1958.

Aristoteles. Aristotelis Analytica Priora et Posteriora. Ed. W. D. Ross. Oxford: Clarendon Press, 1964.

Aristotle. Categories and De Interpretatione. Trans. J. L. Ackrill. Oxford: Clarendon Press, 1963. Aristotle. Prior Analytics. Trans. R. Smith. Indianapolis: Hackett Publishing Company, 1989.

Aristotle. Topics. Books I and VIII. Trans. R. Smith. Oxford: Clarendon Press, 1997.

Aristotle. Prior Analytics. Book I. Trans. G. Striker. Oxford: Clarendon Press, 2009.

Corcoran, J. Completeness of an Ancient Logic. The Journal of Symbolic Logic, Vol. 37, No. 4, pp. 696-702, 1972.

Corcoran, J. A Mathematical Model of Aristotle's Syllogistic. Archiv für Geschichte der Philosophie, Vol. 55, No.2, pp. 191-219, 1973.

Corcoran, J. Aristotle's Natural Deduction System. In: J. Corcoran (ed.). Ancient Logic and its Modern Interpretations. pp. 85-132. Dordrecht: D. Reidel Publishing Company, 1974.

Corcoran, J. Aristotle's Demonstrative Logic. History and Philosophy of Logic, Vol. 30, No. 1, pp. 1-20, 2009.

Crivelli, P. Aristotle's Logic. In: C. Shields (ed.). The Oxford Handbook of Aristotle. Oxford: Oxford University Press, 2012.

Dyckhoff, R. Indirect proof and inversions of syllogisms. Bulletin of Symbolic Logic, Vol. 25, No. 2, pp. 196-207, 2019.

Ferreira, M. R. F. O que são silogismos perfeitos? doispontos, Vol. 10, No. 2, pp. 189-224, 2013.

Malink, M. Aristotle's Modal Syllogistic. Cambridge, MA: Harvard University Press, 2013.

Mignucci, M. Aristotle on the Existential Import of Propositions. Phronesis, Vol. 52, No. 2, pp. 121-138, 2007.

Ockham, G. Opera Philosophica I - Summa Logicae. Ed. P. Boehner, G. Gál and S. Brown. St. Bonaventure, N.Y.: Editiones Instituti Franciscani Universitatis S. Bonaventurae, 1974.

Patzig, G. Aristotle's Theory of the Syllogism: a logico-philological study of book A of the Prior Analytics. Trans. J. Barnes. Dordrecht: Springer Science+Business Media, 1968.

Read, S. Aristotle's Theory of the Assertoric Syllogism. Unpublished, pp. 1-26, 2017, (Available at https://www.st-andrews.ac.uk/ slr/The_Syllogism.pdf).

Smiley, T. J. What Is a Syllogism? Journal of Philosophical Logic, Vol. 2, No. 1, pp. 136-54, 1973.

Smith, R. Logic. In: J. Barnes (ed.). The Cambridge Companion to Aristotle. pp. 27-65. Cambridge: Cambridge University Press, 1995.

von Plato, J. Elements of Logical Reasoning. Cambridge: Cambridge University Press, 2013.

von Plato, J. Aristotle's deductive logic: A proof-theoretical study. In: D. Probst and P. Schuster (eds.). Concepts of Proof in Mathematics, Philosophy, and Computer Science. pp. 323-46. Berlin: De Gruyter, 2016.

von Plato, J. The Great Formal Machinery Works: Theories of Deduction and Computation at the Origins of the Digital Age. Princeton: Princeton University Press, 2017. 